Communication et organisation

42 | 2012

La communication, dimension oubliée de l'intelligence économique

\title{
Compétence de communication, stratégie et intelligence économique en Chine
}

Mylène Hardy

\section{OpenEdition}

\section{Journals}

Édition électronique

URL : http://journals.openedition.org/communicationorganisation/3889

DOI : 10.4000/communicationorganisation.3889

ISSN : 1775-3546

Éditeur

Presses universitaires de Bordeaux

Édition imprimée

Date de publication : 1 décembre 2012

Pagination : 107-124

ISBN : 978-2-86781-772-4

ISSN : $1168-5549$

Référence électronique

Mylène Hardy, « Compétence de communication, stratégie et intelligence économique en Chine »,

Communication et organisation [En ligne], 42 | 2012, mis en ligne le 01 décembre 2014, consulté le 30 avril 2019. URL : http://journals.openedition.org/communicationorganisation/3889 ; DOI : 10.4000/ communicationorganisation.3889 


\title{
Compétence de communication, stratégie et intelligence économique en Chine
}

\author{
Mụlène Hordụ ${ }^{1}$
}

\section{Introduction}

La communication occupe une place centrale dans les activités d'intelligence économique (IE) des entreprises (Moinet, 2009). Les approches positivistes et réductionistes de l'IE comme l'approche économique ont apporté à la construction du concept mais ont leurs limites car, fondées sur la théorie mathématique de l'information, elles négligent de se pencher sur la construction du sens, qui est à la base des activités d'IE, que ce soit dans le questionnement de départ, dans la recherche d'informations, dans l'analyse, etc. En effet, l'information n'est pas un objet, mais se construit en interaction dans une situation donnée (Bulinge \& Agostinelli, 2005), de même que la stratégie de l'entreprise peut être vue comme une construction collective (Burgelman, 1983). Un des moyens de passer d'un modèle informationnel à un modèle communicationnel de l'IE est de se pencher sur la compétence de communication des acteurs de l'IE. Cet angle d'approche permet en effet de prendre en compte les différents types de communication (interpersonnelle, organisationnelle, homme-machine, etc.), en reliant l'acteur construisant l'information avec les autres actants (artefacts, symboles, humains, etc.) qui lui donnent du sens (cf. Leszczynska \& Lesca, 2004), notamment parce que la compétence comprend deux aspects : l'aspect approprié qui fait que l'acteur sait comment communiquer en adéquation avec les règles explicites et implicites de la situation donnée (savoir), et l'aspect stratégique qui fait que l'acteur cherche, et parvient, à atteindre efficacement son but durant et par le biais de l'interaction (savoir-faire). Pour Spitzberg et Cupach (1984), la communication compétente est "l'babileté à s'engager dans une interaction

1 Mylène Hardy est Docteur en Sciences de l'Information et de la Communication, enseignant-chercheur dans plusieurs universités chinoises. Ses recherches portent sur les approches réticulaires en communication des organisations, notamment dans le cadre des activités d'intelligence économique en Chine ; hardymylene@ yahoo.com 
interpersonnelle appropriée et effective $e^{2}$ ( (p. 14). Une part non négligeable des activités d'intelligence économique consiste en la recherche d'informations selon un plan de recherche répondant aux besoins de l'entreprise. Il existe de nombreux supports d'information et donc de nombreux types de sources. Bien que l'information soit de plus en plus codifiée et disponible sur des supports informatiques, une partie des informations ne peut être obtenue que de source humaine (Zhang, 2005). Ceci est d'autant plus vrai dans des pays en transition économique et marqués par l'incertitude, comme la Chine, où le contrôle étatique rend l'accès à l'information ouverte difficile (Hardy \& Agostinelli, 2008); d'où l'intérêt d'étudier la problématique de la recherche d'informations de source humaine dans les activités d'IE en Chine. Tout comme la recherche d'informations électroniques demande un certain nombre de compétences, des compétences de communication sont requises pour recueillir des informations de source humaine. Ces compétences sont construites culturellement à travers la socialisation des acteurs, qui seront ainsi à même de communiquer de manière appropriée. Une autre raison de l'intérêt de la compétence de communication chinoise pour une théorie communicationnelle de l'IE est que, comme le renseignement humain peut s'effectuer efficacement à travers le réseau (Bao et al., 2006), il est utile de se pencher sur la culture chinoise qui met justement le réseau humain au centre de son organisation et de sa communication. Plusieurs études se sont déjà intéressées à la culture chinoise et à ces aspects dans le cadre de l'IE (par exemple, Milliot, 2006) ou plus largement de la stratégie (Fayard, 2009), mais elles restent limitées parce qu'elles utilisent des sources secondaires, ou des sources primaires, mais traduites. Notre étude s'appuie sur une méthode anthropologique basée sur nos notes, enquêtes et entretiens avec des informants directement en chinois ainsi que nos lectures des publications chinoises académiques et grand public. L'avantage de cette méthode inductive est de faire ressortir des points négligés par les études quantitatives sur la culture chinoise. Cet article a donc pour but de poser le cadre théorique de la compétence de communication pour la recherche d'informations de source humaine en Chine, en posant d'abord les principes de la communication chinoise, avant de montrer le lien inhérent entre communication et stratégie en Chine, et de relever les compétences de communication orale utiles dans ce cadre.

\section{Les caractéristiques paradigmatiques de la communication chinoise}

L'on peut, pour définir la communication chinoise, partir dans un premier temps de l'examen de la culture chinoise. De nombreux chercheurs culturalistes $^{3}$ ont tenté d'établir une liste de valeurs proprement chinoises

2 « the ability to engage in appropriate and effective interpersonal interaction".

3 Il nous semble important d'attirer l'attention sur ce point : les études culturalistes, décrivant les grands traits d'une culture en les opposant à une autre, comme Jullien (2003) ou Hofstede (1980) sont utiles pour certains 
(cf. Chinese culture connection, 1987), telles que la piété filiale, l'attachement et la loyauté à la hiérarchie, la recherche de l'harmonie, la modestie ou encore le respect des rites. Linteraction sociale chinoise est caractérisée par la recherche d'harmonie dans le respect de la hiérarchie sociale, suivant une éthique de tradition confucéenne qui met l'accent sur la bienveillance (ren), la justice (yi) et la convenance ou régulation (li) (Gabrenya et Hwang, 1996). Dans le domaine des relations sociales, le li régule le comportement à adopter pour l'interaction en fonction des caractéristiques, notamment du statut social, de son interlocuteur. Les règles d'interaction sont aussi communicatives, la manière dont l'individu s'adresse à l'autre permettant de situer l'un et l'autre dans le système hiérachisé des relations sociales chinoises. En effet, l'individu chinois est ancré dans un réseau de relations interpersonnelles, ou guanxi, codifiées et limitant son action, ce qui fait que la culture chinoise est souvent classée comme une culture collectiviste contraignant l'individu.

La culture chinoise établit une distinction forte entre les membres du réseau et ceux qui sont hors du groupe. Les règles d'interaction, qui comprennent notamment des obligations en termes d'échange de sentiments (renqing), de face (mianzi) et de faveurs (bao), divergent très fortement, nous le verrons, selon ces deux extrêmes. Dans le réseau lui-même cependant, les relations sont hiérarchisées entre les membres les plus proches et ceux plus éloignés. Avec les membres les plus proches, ceux de la famille, il existe un devoir d'entraide, qui s'atténue au fur et à mesure que l'on s'éloigne par le cercle des amis, puis des relations établies par les impératifs de la vie sociale, et enfin des étrangers (Tsui \& Fahr, 1997). De même, la culture chinoise fondant l'interaction sur la proximité relationnelle, l'échange de sentiments et de faveurs, ainsi que les modes communicationnels changent selon le degré de proximité (Gao, Ting-Toomey \& Gudykunst, 1996). Ainsi que le montre le tableau 1, plus l'individu se trouve en face d'un interlocuteur proche relationnellement, plus il a tendance à s'exprimer de manière directe, alors qu'il a recourt à des techniques de communication indirecte et à une forme polie, formelle, de communication dans les autres cas (Gao, Ting-Toomey \& Gudykunst, 1996).

points de la réflexion mais doivent être utilisées avec prudence, car leurs oppositions catégoriques entre les cultures peuvent conduire à des malentendus. La thèse défendue dans cet article est que tous les traits se retrouvent dans toutes les cultures mais y sont plus ou moins mis en avant. 


\begin{tabular}{|c|c|c|c|c|c|c|}
\hline $\begin{array}{l}\text { Type de } \\
\text { relation }\end{array}$ & $\begin{array}{l}\text { Base des } \\
\text { guanxi }\end{array}$ & $\begin{array}{c}\text { Principe } \\
\text { d'interaction }\end{array}$ & Confiance & $\begin{array}{l}\text { Traitement } \\
\text { social }\end{array}$ & Communication & Politesse \\
\hline $\begin{array}{l}\text { Famille } \\
\text { (jiaren) }\end{array}$ & Sang & Obligation & Important & Protection & $\begin{array}{l}\text { Directe / } \\
\text { informelle }\end{array}$ & Moindre \\
\hline $\begin{array}{l}\text { Ami/collègue } \\
\text { (shuren) }\end{array}$ & $\begin{array}{c}\text { Base } \\
\text { commune }\end{array}$ & & & & & \\
\hline $\begin{array}{l}\text { Etranger au } \\
\text { groupe } \\
\text { (shengren) }\end{array}$ & $\begin{array}{l}\text { Aucune } \\
\text { base }\end{array}$ & Utilitarisme & Faible & $\begin{array}{c}\downarrow \\
\text { Suspicion }\end{array}$ & $\begin{array}{l}\text { Indirecte / } \\
\text { formelle }\end{array}$ & Forte \\
\hline
\end{tabular}

Tableau 1 - Relations interpersonnelles et communication en Chine (d'après Chang, 2010 ; Zeng \& Liu, 2004 ; Chen \& Chen, 2004 ; Tsui \& Fahr, 1997 ; Gao, Ting-Toomey \& Gudykunst, 1996 ; Redding, 1993 ; King, 1991/1996)

Les relations seraient en Chine fondées sur le sentiment de proximité, contrairement à la culture occidentale qui mettrait l'accent sur la différence (Zhang Y.H., 2005). Chang et Holt (1991) y voient là aussi une base confucianiste, fondée sur la comparaison de l'autre à soi-même. Ainsi, plus un individu se sent proche d'un autre, plus il pourra partager de sens et construire une base commune pour une communication plus approfondie, ce qui rend la communication chinoise incrémentale, d'où la nécessité d'entendre les guanxi de manière dynamique (Hardy \& Jian, 2011). Chen et Chen (2004) proposent un modèle de développement des guanxi basé sur le commencement des relations, leur construction et leur utilisation. Lors de la première phase, il s'agit de se familiariser avec l'autre pour établir une base commune. La seconde phase consiste à accroître la qualité des relations, évaluée en termes de confiance (xing), de sentiments partagés (ganqing) et de faveurs mutuelles (jiaoqing), lors d'interactions à la fois expressives (participation à des fêtes communes) et instrumentales (soutien mutuel dans les événements de la vie, comme la recherche d'emploi). La dernière phase est une phase instrumentale d'utilisation des relations sur le long terme $e^{4}$. Les relations personnelles sont en Chine considérées comme une ressource sociale dont l'individu tire profit (Chen \& Chen, 2004 ; Chang \& Holt, 1991), et de nombreuses études ont montré que les guanxi étaient utilisés dans les relations commerciales, la recherche d'emploi ainsi que dans la plupart des transactions, le passage par la période communiste n'ayant pas fait disparaitre cette pratique et au contraire l'ayant favorisée, le recours aux relations personnelles servant de moyen de substitution dans un système commercial en transition marqué par l'incertitude (Bao et al., 2006 ; Milliot, 2006 ; Zeng \& Liu, 2004 ; Luo, 2000 ; Xin \& Pearce, 1996 ; Redding, 1993 ; Chang \& Holt, 1991 ; King 1991/1996).

4 Cette instrumentalisation très marquée apparaît nommément dans des expressions courantes, comme «la guanxi » : tirer ses relations, c'est-à-dire y avoir recours. 


\section{L'individualisme dans la communication chinoise}

De ce constat d'instrumentalisation l'on peut comprendre aisément que l'image du Chinois humble et soumis à l'obligation de se maintenir en harmonie avec son réseau de relations en sacrifiant son individualité, reprise dans la plupart des études sur la culture chinoise, ne correspond pas à la réalité des interactions sociales en Chine. "Le fait que les interactants actualisent l'harmonie sociale ne signifie pas qu'ils soient désintéressés ou qu'ils n'aient pas de mobiles ultérieurs" remarque Chang (2001, p. 160). Comme le souligne Chen (2010), la communication chinoise a deux faces, l'une fondée sur l'harmonie du groupe, et l'autre basée sur les jeux de pouvoir. Pour lui, le $l i$ sur lequel se base l'harmonie des relations interpersonnelles gouverne en fait les jeux de pouvoir entre les interactants. Les nombreux exemples existants d'offensive interactionnelle plus ou moins directe selon la situation et le degré de proximité des interactants (cf. Chang, 2010 ; Chang, 2001), peuvent surprendre un observateur qui se serait contenté de lire la liste des valeurs chinoises établies par les chercheurs de la Chinese culture connection (1987) dans laquelle figurent seulement l'attachement à l'harmonie et la noncompétition. L'observation de terrain, loin des seuls questionnaires, montre qu'harmonie et compétition acharnée co-existent, non sans créer des tensions éthiques. Nous en avons observées lorsque l'un de nos informants chinois, praticien de stratégie des entreprises mais aussi des guanxi, nous a offert une année l'ouvrage Houbeixue comme manuel de formation à l'art des guanxi, provoquant une certaine indignation chez nos connaissances communes qui trouvaient cet ouvrage indigne d'être lu à cause de son immoralité. Les interviews que Redding (1993) conduit auprès de managers chinois montrent qu'harmonie confucianiste cohabite avec pouvoir et contrôle 6 . De fait, ces tensions éthiques ont toujours existé : les stratégies de manipulation et de persuasion ont été en général en Chine transmises oralement à cause de leur incompatibilité avec l'éthique confucianiste basée sur l'harmonie (Chen, 2010). Pourtant, la rhétorique et les stratégies liées au langage font partie d'une longue tradition philosophique, politique et littéraire chinoise (Chang, 2010 ; Lu, 2002 ; Jullien, 1995).

\section{Liens entre communication et stratégie en Chine}

Ainsi, la communication chinoise est le lieu d'étude privilégié du double aspect que nous avons soulevé dans la compétence de communication : la poursuite pour l'individu d'une stratégie individuelle tout en se conformant au cadre des contraintes socio-communicationnelles de la situation, dictant

5 Houbeixue, "La doctrine de la peau dure et du coeur noir », est un ouvrage écrit au début du XX $\mathrm{XX}^{\mathrm{e}}$ siècle par "Li Zongwu » qui montre de manière satirique comment avoir pouvoir et fortune en utilisant notamment les guanxi sans considération morale.

6 Un des interviewés dit par exemple «Je pense que les Chinois sont plutôt obsédés par le pouvoir, le pouvoir personnel » ("I think that Chinese are rather obsessed with power, personal power » (Redding, 1993, p. 88)). 
l'harmonie des relations. La communication elle-même est considérée comme une activité stratégique : "Pour les Chinois, la communication peut être perçue comme un outil utilitaire principalement pour obtenir profit et succès dans la société ». (Chang \& Holt, 1991, p. 268). D'ailleurs, les dimensions militaire et lettrée sont inextricablement liées dans la civilisation chinoise (Levi, 2011), et la communication de manipulation et de persuasion chinoise originaire de la tradition chinoise de stratégie militaire continue à être employée dans la vie sociale chinoise contemporaine (Chen, 2010). Chang (2010) distingue trois dimensions dans les pratiques communicationnelles et interactionnelles chinoises : une dimension de manipulation, une dimension artistique et une dimension d'humilité. C'est la dimension de manipulation qui est liée à la stratégie, aussi ne nous attacherons-nous dans cette étude sur la compétence de communication qu'à cette première dimension ${ }^{8}$. D'ailleurs Chang (2010) relie de manière explicite la dimension de manipulation à celle de compétence interpersonnelle et, ce faisant, communicationnelle (aspects stratégique / utilitaire et approprié) dans un système réticulaire. Les fortes contraintes liées à l'aspect approprié de la compétence dans la culture chinoise (respect de la hiérarchie, de la face, de l'harmonie, etc.) obligent le « je » à être plus inventif pour exprimer son individualité que dans des cultures où l'expression individuelle est plus assumée. D'où une forte présence de la communication et des jeux de langage mettant l'accent sur l'aspect créatif et artistique dans les textes de l'antiquité chinoise (cf. Chang, 2010 ; Jullien, 1995). Le lien entre communication et stratégie peut être également expliqué par le courant philosophique chinois du pragmatisme linguistique. Cette tradition confucéenne mais remontant au chamanisme chinois (Thoraval, 2007) veut qu'il y ait conformité entre le langage et le réel : il y a non seulement un aspect approprié disant que la communication doit s'adapter à la situation, mais également un aspect manipulateur du langage pouvant modifier le réel par le pouvoir performatif des mots. Il nous semble que c'est cette croyance qui fonde tout l'aspect de l'utilisation stratégique du langage par le communicateur chinois, dans la tradition comme dans le monde contemporain : bien communiquer, être compétent en communication, c'est-à-dire utiliser le langage de manière stratégique en tenant compte des contraintes situationnelles, permettrait de modifier le système des relations à son avantage. Comme le rappelle Sima Qian, une langue bien affutée permet d'être plus fort que le plus puissant ${ }^{9}$.

\footnotetext{
7 «For the Chinese, communication can be perceived as a utilitarian tool mainly in the case of obtaining profit and succeeding in society"

8 Nous abordons dans notre étude à la fois la manipulation psychologique et la persuasion (la différence portant sur le libre arbitre de l'interlocuteur). Pour Jullien (2002) la tradition chinoise ne considère que la manipulation et non pas la persuasion, qu'il réserve à la tradition occidentale, mais son analyse nous semble trop réductrice. Cheng (1997) montre d'ailleurs l'intensité des débats intellectuels en Chine.

9 “言毛遂至楚，使赵重於九鼎大吕，言为天下所重也.”, Sima Qian (II ${ }^{\mathrm{e}} \mathrm{I}^{\mathrm{er}}$ siècle avant J.C.) , Shiji, 76.

Consulté sur : http://www.guoxue.com/shibu/24shi/shiji/sj_076.htm
} 
Des points communs se retrouvent d'ailleurs dans la conception chinoise de la stratégie comme de la communication : une place privilégiée est en effet accordée à 1) l'aspect circonstanciel, 2) l'aspect processuel, 3) l'aspect combinatoire des choses, 4) à l'information et 5) à la dissimulation.

\section{Les aspects circonstanciel, processuel et combinatoire ou l'intelligence de situation}

Les auteurs de stratégie chinoise relèvent tous l'importance de l'analyse situationnelle, notamment du moment et du terrain, qui doit permettre une meilleure adaptation de l'action. Mao Zedong critique d'ailleurs les stratèges qui tentent d'importer directement en Chine des principes venus d'ailleurs sans se préoccuper d'analyser la situation particulière de la Chine. S'ils établissent bien des distinctions de configurations, les auteurs de stratégie soulignent surtout l'importance de s'adapter aux changements de configuration, et par exemple Sun $\mathrm{Zi}$ conseille de ne pas répéter les tactiques mais de les varier, prenant exemple de l'eau qui n'a pas de forme "régulière ${ }^{10}$ ». Comme le remarque Jullien (1995), la pensée stratégique antique chinoise privilégie la victoire de biais sur celle de front, le biais désignant non seulement ce qui n'est pas ordinaire, mais aussi et surtout la potentialité de l'action. Ce mouvement possible d'une configuration à l'autre s'effectue également selon la direction donnée par le livre des mutations (Yijing). Il s'agit de transformer le faible en fort et inversement, sachant que chaque configuration contient de son opposé latent en elle. Pour Jullien (1995), l'opposition entre rapports de front et de biais dans la stratégie chinoise n'est pas dichotomique, mais mouvante, engendrant ainsi une infinie variation tactique. Il s'agit donc de combiner et non pas d'opposer, tout comme le fait Deng Xiaoping dans sa directive de 1982 : "combiner le civil et le militaire, combiner la paix et la guerre ${ }^{11} »$. Et puisqu'il n'y a pas de limite fixe mais continuité entre les concepts, l'on comprend mieux pourquoi Qiao et Wang (1999) ont plus récemment proposé une réflexion sur la guerre basée sur la combinaison des moyens dans diverses sphères d'action. Leur pensée s'inscrit clairement dans la continuité stratégique chinoise se fondant sur les mutations : "Lorsque l'on décortique son principe [de la guerre entièrement nourvelle], il n'est pas compliqué et s'appuie sur un simple mot: combinaison. "Le Tao engendre l'un, l'un engendre deux, deux engendre trois, trois engendre la multitude": peu importe que ce soit deux, trois ou une multitude, le résultat est toujours la combinaison. Ce n'est qu'avec la combinaison quill y a abondance, quill y a une infinité de changements, quill y a de la diversité. La combinaison a accru les moyens de faire la guerre presque jusqu'à l'infini et a changé la définition de la guerre moderne donnée par le passể ${ }^{12}$ »

10 《故其战胜不复, 而应形于无穷》 《故兵无常势, 水无常形, 能因敌变化而取胜者, 谓之神》, Sunzi bingfa, VI.

11 《军民结合, 平战结合》.

12 《它的原理拆开来看并不复杂, 只是简简单单的两个字 : 组合。“道生一, 一生二, 二生三, 三生 
(p. 131). C'est donc l'intelligence de situation qui permettra au « je » de se mouvoir dans la complexité du réseau combinatoire des facteurs situationnels. Massé, Marcon et Moinet (2007) appellent « intelligence stratégique » cette forme d'intelligence du jeu avec les règles de l'interaction appropriée : «Le réseau est une réalité quil nous appartient d'instrumentaliser pour gouverner le jeu, pour intégrer les règles, identifier et comprendre les acteurs ou encore apprébender la stabilité du jeu afin de le conserver à son avantage, le déstabiliser pour reprendre l'avantage et veiller à ne pas se faire soi-même déstabiliser " (p. 86). Mais il est intéressant de noter qu'en Chine la notion d'intelligence (congming) $)^{13}$ diffère d'une conception analytique et se rapproche plus de la metis grecque. Intégrant l'action, elle est en général associée à celle de stratagème et est parfois connotée négativement : celui qui la possède est malin et, à l'image du Roi singe, parvient à se mouvoir avec adresse dans les situations les plus inextricables. Pour Chen (2010), les stratégies de manipulation et de persuasion chinoises sont liées à la capacité du locuteur à comprendre et s'adapter aux circonstances changeantes tout en maintenant une certaine continuité d'action par l'analyse de la situation temporelle ( $s h i)$, de la situation spatiale (wei) et du début du mouvement (ji). L'intelligence doit selon lui guider l'intégration effective de ces trois éléments dans les stratégies de manipulation. La conception chinoise performative du langage permettrait d'initier le mouvement par la communication, puis de laisser agir pour que la situation évolue en fonction de la stratégie du locuteur selon la vertu de la «propension des choses ", pour reprendre le terme de Jullien (2003). Dans une conversation où les jeux de pouvoir se construisent en situation (Hardy \& Jian, 2011) tout comme dans le jeu de go, l'improvisation, mise en avant dans le $12^{\mathrm{e}}$ des 36 stratagèmes, "Dérober un mouton en profitant de l'opportunité en passant ${ }^{14} "$, prend toute sa place.

\section{L'information et la dissimulation}

L'importance de l'information, constitutive de la communication, est rappelée de manière continue dans les écrits stratégiques chinois, à commencer par Sun $\mathrm{Zi}$ qui en retient aussi bien la dimension offensive que défensive. Dans sa dimension offensive, c'est l'aspect anticipé et basé sur l'humain que Sun Zi souligne ${ }^{15}$. Mais la dimension défensive est tout aussi essentielle, le secret permettant de ménager l'effet de surprise. Ne pas dire, ce peut être aussi dire autrement, d'où la prégnance de la tromperie et de la dissimulation à la fois dans la pensée stratégique et dans la communication chinoise. Les exemples

万物”, 不论二或是三还是万物, 都是组合的结果。组合才有丰富, 组合才有千变万化, 组合才有多 样性。组合使现代战争的手段增至近乎无限, 从根本上改变了既往人们赋予现代战争的定义: 用现 代武器和作战方式进行的战争》.

13 Il existe en chinois plusieurs termes pour traduire la notion d'intelligence.

14 《顺手牵羊》, Shiliu ji, 12.

15 《故明君賢將, 所以動而勝人, 成功出于眾者, 先知也。先知者, 不可取于鬼神, 不可象于事, 不 可驗于度。必取于人, 知敵之情者也。”Sunzi bingfa, XIII. 
de stratagèmes destinés à tromper l'adversaire ne manquent pas dans les écrits stratégiques chinois, certains des 36 stratagèmes étant significatifs : faire du bruit à l'est et attaquer à l'ouest, inventer là où il n'y a rien, cacher un couteau dans son sourire, voler la poutre et la remplacer par le pilier, montrer le mûrier et insulter le sophora, et faire semblant d'être un idiot sans être fou $^{16}$. Ces stratagèmes sont entrés dans la sagesse populaire et font partie des nombreux proverbes chinois sur la communication. La performativité du langage prend là encore toute sa place, puisque "la désinformation fait passer des cartes, soit des systèmes finalisés d'interprétation, pour la réalité des territoires qu'elles décrivent de manière orientée afin que ce qui est dit l'emporte sur ce qui est» (Fayard, 2009, p. 19).

La stratégie en 24 caractères de Deng Xiaoping des années 90 reflète là encore bien une continuité stratégique et communicationnelle de l'acteur chinois observateur des règles situationnelles d'interaction, des jeux de pouvoir, modeste pour l'apparence de la communication appropriée et harmonieuse, mais ayant sa propre stratégie qu'il dissimule à son interlocuteur ${ }^{17}$. Ainsi, la communication chinoise peut se résumer par cette citation datant de l'époque des Song et passée dans la sagesse populaire : xianwai zhiyin ${ }^{18}$, le son émis au-delà de la corde, autrement dit le double sens du langage. Le principe interactionnel chinois se fonde sur la recherche de l'harmonie des relations, mais aussi sur la possibilité d'attaques dans un sens ou dans l'autre, d'où une grande prudence vis-à-vis des informations que l'on donne et l'utilisation de stratégies indirectes pour garder la face tout en se ménageant des possibilités de jeu, au sens spatial et ludique du terme, pour garder la potentialité de l'action (Hardy, 2008) ${ }^{19}$.

\section{La compétence de communication dans les activités orales de recherche d'information}

Pour pouvoir communiquer de manière effective, il faut connaître le contexte culturel dans lequel les règles de communication sont construites. Chen (2010) rappelle cinq principes de stratégie d'interaction passés dans la sagesse populaire chinoise et utilisés en communication: 1) appréhender la situation dans sa globalité ; 2) maximiser les intérêts potentiels ; 3) être flexible ; 4) être prudent et 5) garder les secrets. Celui qui parle le premier périt, remarquent Zeng et Liu (2004). C'est pourquoi le principe premier mis

16 6. 声东击西、 7. 无中生有、10. 笑里藏刀、25. 偷梁换柱、26. 指桑骂槐、27. 假痴不颠, Sanshiliu ji. 17 冷静观察, 稳住阵脚, 沉着应付, 蹈光养晦, 善于守拙, 决不当头. Observer calmement, sécuriser les positions, faire face posément, dissimuler ses capacités, attendre le bon moment, maintenir un profil bas et ne surtout pas clamer sa supériorité.

18 弦外之音

19 Cela ne signifie pas, naturellement, que la communication chinoise ne soit jamais directe : au contraire elle comprend des stratégies directes et indirectes au même titre que la communication occidentale. La différence cependant est une conceptualisation des stratégies indirectes plus détaillée, qui en rendent la lecture intéressante pour renouveler les théories communicationnelles actuelles basées sur des prémisses occidentales. 
en avant dans les manuels grand public de communication en Chine ainsi que dans la socialisation des plus jeunes n'est pas de parler mais d'écouter. Un de ces manuels (Liu, 2009) débute par une partie sur « deux grandes oreilles pour écouter » en donnant l'exemple d'un commerçant du XIX de passage à Shanghai qui s'enrichit grâce à l'exploitation intelligente d'une information entendue dans un salon de thé. Le manuel donne de nombreux conseils d'écoute active, et cite comme bien d'autres Confucius : «En se promenant à trois, chacun trouvera certainement un maître parmi les autres ${ }^{20} "$.

Ecouter permet également de mieux comprendre l'autre et de s'y adapter. Premièrement, cela permet d'interpréter le sens du discours. En effet, dans le cadre d'une conversation courante, plus l'interlocuteur s'éloigne du cercle familial et amical du « je ", moins la confiance est accordée et plus celui qui parle a recourt à un style indirect si le sens qu'il souhaite convoyer entre en contradiction avec les principes de la communication appropriée. L'empathie, dont nous avions vu qu'elle était nécessaire à l'établissement des guanxi, est donc nécessaire pour déceler le sens caché et interpréter correctement le message. L'empathie est d'ailleurs une des qualités de locuteur chinois dans son aspect modeste : le " je » fait toujours passer l'autre avant lui (Chang, 2010). Mais cette règle de la communication appropriée chinoise, qui veut que le « je » loue la connaissance de l'autre et dénigre la sienne, peut aussi être avantageusement utilisée par le locuteur stratège pour recueillir des informations (cf. stratagème 27 plus haut).

Écouter permet également de mieux s'adapter à son interlocuteur dans un but manipulatoire, dimension de la communication traditionnelle chinoise rappelée par Guiguzi $i^{21}$, qui préconise de parler de manière érudite avec les personnes intelligentes, de manière modeste avec les gens bornés, etc. Cet aspect de la compétence de communication montre que la culture chinoise ne met pas en avant la face, mais des faces, et que l'intelligence manipulatrice est associée avec la capacité du locuteur de se positionner selon les différentes faces en étant comme le dit un proverbe " agile à huit faces ${ }^{22}$ ". Ce type de proverbe a une connotation négative en Chine. Mais le travail de la face, pour reprendre Goffman (1972), ne doit pas nécessairement faire penser qu'il faut se dissimuler entièrement sous un autre personnage. Pour la tradition confucéenne chinoise, la construction des guanxi qui permettent de recueillir l'information devrait s'effectuer avec sincérité : «Pour converser il faut se faire des amis, pour se faire des amis il faut ouvrir son coeur et traiter les autres avec

20 三人行, 必有我师焉.Lunyu, VII.

21 Guiguzi, traité de stratégie diplomatique et politique attribué à Wang Xu (IVe siècle avant J.C.), mais vraisemblablement écrit plus tard. Récemment remis à l'honneur dans les traités de communication grand public.

22 八面玲珑 
sincérite $e^{23}$ "(Zhang, 2005, p. 159) ${ }^{24}$, position qui n'est pas partagée par tous les spécialistes (cf. Chen, 2011). À travers ce parallèle effectué par Zhang (2005) entre l'échange de paroles (jiaotan), l'échange d'amis (jiaoyou) et l'échange des sentiments (jiaoxin), l'on voit comment la communication chinoise, fortement contextualisée, mêle inextricablement le contenu du message à l'échange quasi physique des sentiments. Cela a plusieurs incidences dans le cadre de recherche orale d'informations (Hardy, 2008) : 1). Tout d'abord, le type de communication à privilégier doit être le face-à-face, car les autres types de communication ne permettent pas une aussi grande richesse des sentiments et de l'échange. Comme nous l'ont dit des informants, les techniques de recherche d'informations téléphonique préconisées aux États-Unis sont difficiles à mettre en place en Chine à cause du fort soupçon pesant sur les intentions des interlocuteurs inconnus, qui renforce la culture du secret. Or plus la communication est riche (plusieurs canaux de communication simultanés), plus les interlocuteurs pourront se jauger et décider du degré de secret à garder. Et plus l'empathie est grande, plus les informations échangées seront riches. 2) Pour permettre une plus grande empathie, les règles de construction des guanxi veulent que l'on commence d'abord par mettre en place des "bases de guanxi", puis développer peu à peu les relations, ce qui prend du temps (Chen \& Chen, 2004). C'est pourquoi Zhang (2005) explique que la réussite du recueil d'information de source humaine se trouve dans l'expérience sociale des agents de renseignement ${ }^{25}$, dans leur capacité à se socialiser et dans leur sensibilité au sens de l'information. La capacité de socialisation est essentielle, car plus les guanxi seront développés, plus il y aura de confiance et plus l'information échangée sera riche. Zhang (2005) juge par ailleurs les cadres supérieurs être de meilleurs agents de renseignement que les autres car ils ont accès à plus de monde que des employés même spécialisés dans l'intelligence économique. Ce trait est dû à la forte hiérarchisation des relations sociales qui fait que l'information, très cloisonnée et restant au plus haut niveau de la hiérarchie de l'entreprise, ne peut être atteinte que par des relations initiées par des gens d'un niveau plus ou moins égal (cf. Hardy, 2008). Chen (2011) recommande d'ailleurs de passer par plusieurs niveaux de relations pour parvenir à toucher les personnes qui détiennent l'information recherchée. Cela est rendu possible par l'aspect transactionnel des guanxi : la confiance est transférée par l'intermédiaire qui permet à deux nouvelles personnes de se rencontrer (Chang, 2010). Par ailleurs, plus les guanxi sont

23 《交谈必须交友, 交友必须交心, 以诚待人》.

24 Il existe des dizaines d'ouvrages en Chine traitant de l'intelligence économique pour les entreprises, mais la plupart restent théoriques. Nous avons sélectionné pour cet article deux ouvrages écrits par des praticiens afin de rendre compte des pratiques de terrain (Chen, 2011; Zhang, 2005), confirmées par notre enquête sur les pratiques des cadres intermédiaires de deux PME chinoises (cf. Hardy, 2008). Il faut noter cependant que ce que les auteurs d'ouvrages écrivent ne reflète pas nécessairement toujours leur pratique.

25 Nous traduisons par « agent de renseignement » le terme chinois «情报人员 » mais ce terme pourrait aussi être rendu par « employé d'intelligence » et doit être compris dans son sens large. 
développés, plus les interlocuteurs se situent dans un cercle proche et plus la communication sera directe, ce qui permet une interprétation plus facile du sens du message. Une communication directe ne signifie pas de déroger aux règles de la communication appropriée que nous avons citées plus haut, et d'ailleurs Chen (2011) rappelle que la caractéristique de l'intelligence est la dissimulation et recommande de ne pas dévoiler le vrai sujet de la conversation même avec une relation proche. Lors de l'interaction, il convient donc d'utiliser des modes de communication implicites qui permettent à la fois de préserver la face de chacun, l'agenda de chacun et une place suffisante pour la négociation (Gao, Ting-Toomey \& Gudykunst, 1996).

Le tableau 2 s'appuie sur la littérature sur la compétence de communication (cf. Hardy, 2008) et détaille les compétences de communication qui doivent être actionnées pour un recueil efficace d'informations de source humaine en Chine. Ces compétences sont principalement actionnables dans le cadre de la mise en place d'un réseau d'experts et de ressources tel qu'il a pu être préconisé par Bao et al. (2005).

\begin{tabular}{c|c}
\hline $\begin{array}{c}\text { Type de compétence de } \\
\text { communication }\end{array}$ & Compétence à actionner \\
\hline Gestion de la relation & Construire les guanxi \\
& $\begin{array}{c}\text { mise en place d'une base de guanxi } \\
\text { échange de faveurs et de sentiments } \\
\text { construction de la relation sur le long terme } \\
\text { Installer le contexte d'une communication riche } \\
\text { Choisir une situation de communication } \\
\text { informelle } \\
\text { Préférer le face-à-face }\end{array}$ \\
\hline Complexité cognitive & Analyser les éléments de la situation \\
Lieu, moment, opportunité
\end{tabular}




\begin{tabular}{c|c} 
Utiliser la communication indirecte pour : \\
- Ne pas dévoiler ses objectifs \\
- Préserver l'information \\
- Laisser à l'autre une ouverture \\
Utiliser la situation de l'échange de manière \\
effective et constructive \\
- Construire le sens à deux \\
- Influencer la construction du sens \\
Savoir-être & Faire en sorte que la situation évolue selon le \\
schéma d'action construit par la conversation
\end{tabular}

Tableau 2 - Compétences de communication pour le recueil d'informations de source humaine en contexte chinois

\section{Conclusion}

La communication chinoise, qu'elle soit interpersonnelle ou organisationnelle, produit des règles d'interaction spécifiques, dont notre article n'a abordé que les principes essentiels. Une recherche de type exploratoire comme la nôtre a l'avantage de sonder les principes culturels en profondeur, mais possède l'inconvénient de la lenteur et du manque de systématisation inhérent à toute approche anthropologique. Il conviendra donc pour la recherche future de systématiser les stratégies de communication utilisées et de mettre en place un protocole de recherche quantitative pour en vérifier la fiabilité. Il serait par ailleurs utile de relier de manière plus précise les stratégies avec les types de situation de recherche d'information, là encore dans un but prototytique. Enfin, notre article laisse en suspens deux questions qu'il serait intéressant d'explorer : le lien entre communication/stratégie individuelle, et communication/stratégie organisationnelle, et la question de la construction des compétences dans l'entreprise.

La double face de la communication chinoise, entre le respect règles collectives et la place laissée à la stratégie individuelle, correspond au double aspect de la compétence de communication, et permet également de faire le lien entre micro et macro, entre communication interpersonnelle et communication organisationnelle, entre compétence individuelle et compétence organisationnelle. Dans le cadre des activités d'intelligence économique, la question du lien individu / organisation est cruciale pour 
permettre une construction collective du plan de renseignement avant la recherche d'information et une diffusion effective de l'information recueillie dans l'organisation, ou plutôt une réelle construction collective du sens à partir des informations recueillies. Les compétences de communication nécessaires aux différentes phases du cycle d'IE ne doivent pas être de simples connaissances ou de dispositions, mais doivent pouvoir être actionnées en situation. Aussi s'agit-il, dans le cadre d'une réflexion sur les ressources humaines, de construire ces compétences sur le long terme, tout comme les guanxi. Chen (2011) propose par exemple une approche dynamique de la communication dans le cadre des activités d'intelligence économique, qui permet à l'agent de renseignement de passer par le stade de la copie d'information ouverte trouvée dans les médias, à celui de l'écoute, puis à celui de la discussion permettant d'aborder plusieurs sujets à enfin celui du questionnement actif et habile.

Notre article s'inscrit dans une perspective transculturelle : loin de simplement décrire des spécificités culturelles propres à la Chine, nous avons souhaité montrer les concepts qui, dans cette culture, semblaient intéressants à utiliser pour une meilleure appréhension de la communication dans l'intelligence économique, justement parce que la culture chinoise met certains d'entre eux plus en avant que d'autres cultures. Il est d'ailleurs à remarquer que les chercheurs chinois en IE utilisent quasiment exclusivement des concepts occidentaux sans s'interroger sur les représentations chinoises (cf. Bao et al., 2006), même si le regain d'intérêt pour la culture traditionnelle en Chine devrait changer cette tendance. L'enrichissement de la recherche doit s'effectuer de manière non seulement transculturelle, mais aussi de manière interdisciplinaire. Dans ce domaine de la construction des compétences comme dans d'autres, il semble que les réflexions sur la communication dans les activités d'intelligence économique peuvent utilement s'appuyer sur celles déjà en cours dans d'autres domaines connexes, comme le renseignement d'Etat, la recherche scientifique, le journalisme, la diplomatie ou la négociation.

\section{BIBLIOGRAPHIE}

BAO C. et al., « Renji qingbao wangluo », Qingbao lilun yu shijian, (2), 2006, 129-141.

BULINGE F., AGOSTINELLI S., «L'analyse d'information : d'un modèle individuel à une culture collective ", R3I, 2005, p. 1-18.

BURGELMAN R. A., " A model of the interaction of strategic behavior, corporate context, and the concept of strategy ", Academy of Management Review, (8) 2, 1983, p. 61-70.

CHANG H.-C., Clever, creative, modest: The Chinese language practice, Shanghai, Shanghai foreign language education press, 2010, $294 \mathrm{p}$. 
CHANG H.-C., "Harmony as performance : the turbulence under Chinese interpersonal communication », Discourse Studies, 3, 2001, p. 155-179.

CHANG H.-C., HOLT G. R., "More than relationship : Chinese interaction and the principle of kuan-bsi », Communication Quarterly, 39 (3), 1991, p. 251-271.

CHEN B., Qiye qingbao shiwu, Shanghai, Wenhui chubanshe, 2011, 263 p.

CHEN G., Study on Chinese communication behaviors, Hong-Kong, China Review Academic publishers, 2010, 313 p.

CHEN X., CHEN C. C., "On the intricacies of the Chinese guanxi: A process model of guanxi development ", Asia Pacific Journal of Management, 21, 2004, p. 305-324.

CHENG A., Histoire de la pensée chinoise, Paris, Seuil, 1997, 696 p.

CHINESE CULTURE CONNECTION, "Chinese values and the search for culture-free dimensions of culture ", Journal of Cross-cultural Psychology, 18(2), 1987, p. 143-164.

FAYARD P., Sun Tzu : stratégie et séduction, Paris, Dunod, 2009, 205 p.

GABRENYA W. K., HWANG K.-K., " Chinese Social Interaction: Harmony and Hierarchy on the Good Earth ", in BOND M. H. (ed), The Handbook of Chinese Psychology, Hong-Kong, Oxford University Press, 1996, p. 309-321.

GAO G., TING-TOOMEY S., GUDYKUNST W. B., « Chinese Communication Processes ", in Mickael H. BOND (ed), The Handbook of Chinese Psychology, Hong-Kong, Oxford University Press, 1996, 282-293 p.

GOFFMAN E., «On-Face Work: An analysis of ritual elements in social interaction », in GOFFMAN E., Interaction ritual : Essays on face-to-face behaviour, Harmondsworth, Penguin, 1972, p. 5-45.

HARDY M., JIAN G., « Materializing guanxi: Exploring the communicative practice of liao tian in Chinese business settings ", in KUHN T. (ed.), Matters of Communication: Political, Cultural, and Technological Challenges to Communication Theorizing, New York, Hampton Press, 2011, p. 167-195.

HARDY M., AGOSTINELLI S., « La dimension culturelle des pratiques communicationnelles de veille stratégique et son influence sur les systèmes de veille : l'exemple des entreprises chinoises », in FLORY A., GHENIMA M. et SIDHOM S. (eds.), Système d'information et intelligence économique, Tunis, IHE éditions, 2008, p. 249-262.

HARDY M., De l'intelligence informationnelle à l'intelligence communicationnelle : la compétence communicationnelle des cadres intermédiaires dans les processus d'intelligence économique. Le cas de deux PME chinoises, Thèse de doctorat, Université d'Aix-Marseille 3, 2008.

HOFSTEDE G., Culture's Consequences: International Differences in Work-Related Values, Beverly Hills, Sage, 1980, 475 p.

JULLIEN F., Le Détour et l'Accès, stratégies du sens en Chine, en Grèce, Paris, Grasset, 1995, 462 p.

JULLIEN F., La Propension des choses, pour une histoire de l'efficacité en Chine, Paris, Seuil, 1992, 282 p. ; réed. 2003

JULLIEN F., Traité de l'efficacité, Paris, Grasset, 1996/2002, 240 p. 
KING Y. C., "Kuan-hsi and network building : A sociological interpretation ", in BROWN R. A. (Ed.), Chinese Business Entreprise vol. II, London, Routledge, 1996, p. 321-338, (Original work published in 1991).

LESZCZYNSKA D., LESCA H., «Veille Stratégique : utilité des "informations de terrain" pour la PME-PMI à la recherche de nouveaux débouchés produit/marché, étude d'un cas ", Papier de Recherche CERAG UMR 5820 CNRS, 2004. Consulté sur http:// www.veille-strategique.org

LÉVI J., Réflexions chinoises : lettrés, stratèges et excentriques de Chine, Paris, Albin Michel, 2011, 253 p.

LIU X., Goutong jiqiao - zhongguoren de jiaoji zhihui, Shenyang, Wanjuan chuban gongsi ; 2009, $400 \mathrm{p}$.

LU X., "Comparative studies in Chinese and Western rhetorics: reflections and challenges », in JIA W., LU X., D. HEISEY R. (ed.), Chinese Communication Theory and Research: Reflections, New Frontiers and New Directions, Westport, Greenwood, 2002, p. 105-120.

LUO Y., Guanxi and business, Singapore, World Scientific, 2000, 342 p.

MASSÉ G., MARCON C., MOINET N., « Les fondements de l'intelligence économique : Réseaux \& jeu d'influence », Market Management, (2)3, 2006, p. 84-103.

MILLIOT É., « L'intelligence économique dans un pays à contexte culturel fort : cas de la République Populaire de Chine ", Market Management, (2)3, 2006, p. 72-83.

MOINET N., "L'épistémologie de l'intelligence économique face au défi de la communication ", Revue Internationale d'intelligence économique, (1), 2009, p. 159-173.

QIAO L., WANG X., Chaoxian zhan, Beijing, jiefangjun wenyi chubanshe, 1999, 254 p.

REDDING G., The spirit of Chinese capitalism, Berlin, De Gruyter, 1993, 269 p.

SPITZBERG B. H., CUPACH W. .R., Interpersonal Communication Competence, Beverly Hills, Sage, 1984, 247 p.

THORAVAL J., "La tentation pragmatique dans la Chine contemporaine ", in CHENG A. (dir.), La pensée en Chine aujourd'hui, Paris, Gallimard, 2007, p. 103-134.

TSUI A. S., FARH J.-L. L., "Where guanxi matters: Relational demography and guanxi in the Chinese context ", Work and Occupations, 24(1), 1997, p. 56-79.

XIN K. K., PEARCE J. L., " Guanxi: Connections as substitutes for formal institutional support ", Academy of Management Journal, 39(6), 1996, p. 1641-1658.

ZENG S., LIU J., Renji guanxi yu goutong, Beijing, Qinghua daxue chubanshe, 2004, $271 \mathrm{p}$.

ZHANG S., Jingji qingbao jueding qiye mingyun, Beijing, Zhongguo fazhan chubanshe, 2005, 365 p.

ZHANG Y., "Guanxi: yige bentu zuzhi xinweixue yanjiu fanshi de goujian ", Neimenggu shehui kexue, 26 (4), 2005, p. 118-122.

Résumé : Cet article propose un cadre théorique pour l'étude des compétences de communication mises en place dans les activités d'intelligence économique en Chine, en particulier dans le cadre du recueil d'informations de source humaine et l'utilisation du 
réseau de relations interpersonnelles. Basée sur une approche anthropologique et un terrain de plusieurs années, cette recherche part de la conception chinoise de la communication et de la stratégie pour en définir les liens et montrer comment le communicateur chinois se positionne comme stratège dans la construction et l'utilisation de son réseau social. À partir des stratégies ainsi définies, l'article développe un cadre de compétences de communication actionnables en situation de recueil d'informations orales.

Mots-clés : Compétence de communication, communication chinoise, stratégie chinoise, intelligence économique, recueil d'informations de source humaine.

Abstract : This paper aims to build a theoretical framework for the study of the communication compentencies needed in competitive intelligence activities in China, with a focus on information collection from human sources and the use of interpersonal networks. It studies the Chinese conception of communication and strategy as well as their relationships from an anthropological approach that is based on a field work carried out over several years. The research examines how the Chinese communicator positions him/herself as a strategist during the construction and the use of his/her social network and it defines these strategies. A framerwork for communication competencies is then proposed that can be implemented to collect information from oral sources.

Keywords : Communicative competence, Chinese communication, Chinese strategy, competitive intelligence, bumint. 
